\title{
Newton-Raphson based modified Laplace Adomian decomposition method for solving quadratic Riccati differential equations
}

\author{
Vinod Mishra ${ }^{1}$ and Dimple Rani ${ }^{1, \text { a }}$ \\ ${ }^{1}$ Department of Mathematics, Sant Longowal Institute of Engineering and Technology, Longowal, Punjab, India
}

\begin{abstract}
Numerical Laplace transform method is applied to approximate the solution of nonlinear (quadratic) Riccati differential equations mingled with Adomian decomposition method. A new technique is proposed in this work by reintroducing the unknown function in Adomian polynomial with that of well known NewtonRaphson formula. The solutions obtained by the iterative algorithm are exhibited in an infinite series. The simplicity and efficacy of method is manifested with some examples in which comparisons are made among the exact solutions, ADM (Adomian decomposition method), HPM (Homotopy perturbation method), Taylor series method and the proposed scheme.
\end{abstract}

\section{Introduction}

One of the most significant classes of nonlinear differential equation, Riccati differential equation (RDE) is considered as follows:

$$
\frac{d y}{d t}=q(t) y+r(t) y^{2}+p(t), y(0)=a
$$

where $q(t), r(t)$ and $p(t)$ are the known scalar functions and $a$ is an arbitrary constant. The RDE is named after the name of Italian nobleman Count Jacopo Francesco Riccati(1676-1754) [4]. In the field of applied science and engineering, the RDEs have played an essential role $[2-7,29]$. A one dimensional static Schrodinger equation is closely related to RDEs. Satisfying projective Riccati equations, solitary wave solutions of nonlinear partial differential equations can be expressed as polynomials in two elementary functions [4, 7]. In some control theory problems such as dynamic games, linear system with Markovian jumps and stochastic controls RDEs act predominantly [7,29]. Apart from these applications RDEs is also used in stochastic realization theory, robust stabilization, and network synthesis and presently in financial mathematics [4]. Much attention has been given to solve these kinds of equations due to the above applications.

Certain methods are there in literature to solve the RDEs. In [2], Ghorbani and Momani applied the piecewise variational iteration method (VIM) to solve the RDEs. Differential transform method $[4,9]$ is adopted to find the solution of RDEs. Taiwo and Osilagun [5] approximated the solution of RDEs by Iterative algorithm. Perturbation iteration algorithm (PIA) has been presented in solving RDEs [8]. Vahidi has made the comparison among HPM,
ADM and LTDM in solving RDEs in [3]. For solving these kinds of equations Yang et.al. [7] employed the hybrid functions and Tau method. In [6] the authors developed the iterative methods ADM, MADM, VIM, MVIM, HPM, MHPM and HAM to solve the general RDE.

Laplace transform is a powerful tool in solving linear problems but it is incapable of solving nonlinear problems. A well known numerical algorithm Laplace transforms and Adomian decomposition method has conquered much importance in solving many linear and nonlinear problems which provides a series solution. Suheil A. Khuri was the first to apply Laplace decomposition algorithm to solve a class of nonlinear differential equation [12]. A combined Laplace Adomian decomposition method is used to solve nonlinear Volterra integral equation with weakly kernel [1]. In [10], Majid Khan et. al. solved nonlinear coupled partial differential equations with the help of Laplace Decomposition method. LDM is also implemented to obtain the series solution of nonlinear fractional differential equations [18]. Waleed Al-Hyani [22] solved nth order Integrodifferential equations by the usage of LT-ADM. In [14], Modified Laplace decomposition method is proposed for solving Lane-Emden type differential equation. LDM is exercised to solve the Logistic differential equations in [11]. Wazwaz [27] employed CLT-ADM for solving nonlinear volterra-integro differential equations. For handling the solutions of nonlinear system of partial differential equation Laplace decomposition method and pade approximant is used in [21]. Hence this method is utilized to solve many more problems like Singular initial value problems [13], Double singular boundary value 
problems [15], Higher order boundary value problems [16]. Other cited references are [17, 19, 20, 23-26].

In the proposed numerical technique, we use the Laplace transform-Adomian decomposition method to solve the Quadratic RDEs. The main thrust of this methodology is to replace the unknown function $y_{i}$ in Adomian polynomial with Newton-Raphson formula, which improves the Adomian polynomial. The outline of this paper is as follows: Section 1 contains a brief summary on RDEs and Laplace transform combined with Adomian decomposition method. In Section 2, we developed the method based on LT-ADM. In Section 3, the numerical results of employing the technique on three examples for RDEs are presented. In the end conclusions are drawn.

\section{Analysis of Method}

For finding the solution of Quadratic RDE with initial condition is given by equation (1). The method consists of applying the Laplace transform on both sides of (1),

$$
L\left[\frac{d y}{d t}\right]=L\left[q(t) y+r(t) y^{2}+p(t)\right]
$$

Applying the linear and differential property of Laplace transform,

$$
L[y]=\frac{a}{s}+\frac{1}{s} L[q(t) y]+\frac{1}{s} L\left[r(t) y^{2}\right]+\frac{1}{s} L[p(t)]
$$

In this method the solutions are presented as an infinite series given by

$$
y=\sum_{n=0}^{\infty} y_{n}
$$

where the term $y_{n}$ are to be computed recursively and the nonlinear term $f(y)=y^{2}$ is decomposed as follows:

$$
f(y)=y^{2}=\sum_{n=0}^{\infty} A_{n}
$$

here $A_{n}=\frac{1}{n !} \frac{d^{n}}{d \lambda^{n}}\left[f \sum_{i=0}^{n} \lambda^{i}\left(y_{i}-\frac{f\left(y_{i}\right)}{f^{\prime}\left(y_{i}\right)}\right)\right]_{\lambda=0}$

A few terms of $A_{n}$ are given by

$$
\begin{gathered}
A_{0}=\left(\frac{1}{2}\right)^{2} y_{0}{ }^{2} \\
A_{1}=\left(\frac{1}{2}\right)^{2} 2 y_{0} y_{1} \\
A_{2}=\left(\frac{1}{2}\right)^{2}\left(2 y_{0} y_{2}+y_{1}{ }^{2}\right)
\end{gathered}
$$

Using (3) and (4) in (2) and by using the linearity property of Laplace transform, we obtain

$$
\sum_{n=0}^{\infty} L\left[y_{n}\right]=\frac{a}{s}+\frac{1}{s} \sum_{n=0}^{\infty} L\left[q(t) y_{n}\right]+\frac{1}{s} \sum_{n=0}^{\infty} L\left[r(t) A_{n}\right]+\frac{1}{s} L[p(t)]
$$

Comparing both sides we have the following recursive relation

$$
\begin{gathered}
L\left[y_{0}\right]=\frac{a}{s}+\frac{1}{s} L[p(t)] \\
L\left[y_{1}\right]=\frac{1}{s} L\left[q(t) y_{0}\right]+\frac{1}{s} L\left[r(t) A_{0}\right] \\
L\left[y_{2}\right]=\frac{1}{s} L\left[q(t) y_{1}\right]+\frac{1}{s} L\left[r(t) A_{1}\right]
\end{gathered}
$$

In general, the relation is given by

$$
L\left[y_{n+1}\right]=\frac{1}{s} L\left[q(t) y_{n}\right]+\frac{1}{s} L\left[r(t) A_{n}\right]
$$

To get the values of $y_{0}, y_{1}, y_{2}, y_{3}, \ldots$, apply the inverse Laplace transform to above equations

$$
y_{0}=L^{-1}\left[\frac{a}{s}+\frac{1}{s} L[p(t)]\right]
$$

Substitute the value of $y_{0}$ into (8), evaluate the value the R.H.S. and then take inverse Laplace transform will give the value of $y_{1}$. In this manner we get the terms $y_{2} y_{3} \ldots$ recursively and by putting these values in (3) give the solution to the problem.

\section{Numerical Results}

In this section we employ our method to different forms of Quadratic RDE.

Example 1.Consider the following [8]

$$
y^{\prime}=1+y^{2}(t), y(0)=0
$$

Which has the exact solution as $y=\tan t$.

The solution at the different values of $t$ is found and presented through Table1. From Table 1 it can be seen that the solution obtained by our method is very much close to the exact solution and Figureldemonstrates the superiority of our method.

Table 1. Computed exact and approximate solution for Example1

\begin{tabular}{|c|c|c|c|}
\hline $\mathbf{t}$ & Exact & $\begin{array}{c}\text { Approximate } \\
\text { solution }\end{array}$ & Absolute Error \\
\hline 0 & 0 & 0 & $0.0000000 \mathrm{E}+00$ \\
\hline 0.01 & 0.010000333 & 0.010000083 & $2.5001250 \mathrm{E}-07$ \\
\hline 0.02 & 0.020002667 & 0.020000667 & $2.0004001 \mathrm{E}-06$ \\
\hline 0.03 & 0.030009003 & 0.03000225 & $6.7530387 \mathrm{E}-06$ \\
\hline 0.04 & 0.040021347 & 0.040005334 & $1.6012809 \mathrm{E}-05$ \\
\hline 0.05 & 0.050041708 & 0.050010419 & $3.1289104 \mathrm{E}-05$ \\
\hline 0.06 & 0.060072104 & 0.060018006 & $5.4097349 \mathrm{E}-05$ \\
\hline 0.07 & 0.070114558 & 0.070028597 & $8.5960526 \mathrm{E}-05$ \\
\hline 0.08 & 0.080171105 & 0.080042694 & $1.2841072 \mathrm{E}-04$ \\
\hline 0.09 & 0.090243790 & 0.090060799 & $1.8299066 \mathrm{E}-04$ \\
\hline 0.1 & 0.100334672 & 0.100083417 & $2.5125533 \mathrm{E}-04$ \\
\hline
\end{tabular}




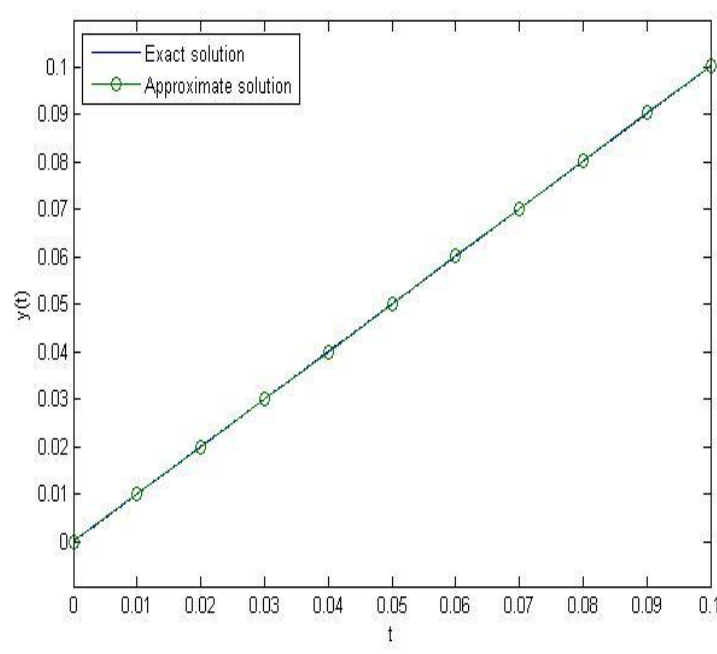

Figure 1. Comparaison of exact and approximate solution for Example1.

Example2. Consider the another type of Quadratic RDE $[4,5,7]$

$$
y^{\prime}=1+y^{2}(t), y(0)=0
$$

which has the exact solution as $y=\frac{e^{2 t}-1}{e^{2 t}+1}$.

According to the above methodology we attain the following results:

Table 2. Computed exact and approximation solution for Example2

\begin{tabular}{|c|c|c|c|}
\hline $\mathbf{t}$ & $\begin{array}{c}\text { Exact } \\
\text { solution }\end{array}$ & $\begin{array}{c}\text { Approximate } \\
\text { solution }\end{array}$ & Absolute error \\
\hline 0 & 0 & 0 & $0.0000000 \mathrm{E}+00$ \\
\hline 0.01 & 0.009999667 & 0.009999917 & $2.4998750 \mathrm{E}-07$ \\
\hline 0.02 & 0.019997334 & 0.019999333 & $1.9996001 \mathrm{E}-06$ \\
\hline 0.03 & 0.029991003 & 0.02999775 & $6.7469637 \mathrm{E}-06$ \\
\hline 0.04 & 0.03997868 & 0.039994668 & $1.5987209 \mathrm{E}-05$ \\
\hline 0.05 & 0.049958375 & 0.049989586 & $3.1210979 \mathrm{E}-05$ \\
\hline 0.06 & 0.059928104 & 0.059982006 & $5.3902948 \mathrm{E}-05$ \\
\hline 0.07 & 0.06988589 & 0.069971431 & $8.5540349 \mathrm{E}-05$ \\
\hline 0.08 & 0.079829769 & 0.079957361 & $1.2759151 \mathrm{E}-04$ \\
\hline 0.09 & 0.089757785 & 0.089939299 & $1.8151442 \mathrm{E}-04$ \\
\hline 0.1 & 0.099667995 & 0.09991675 & $2.4875529 \mathrm{E}-04$ \\
\hline
\end{tabular}

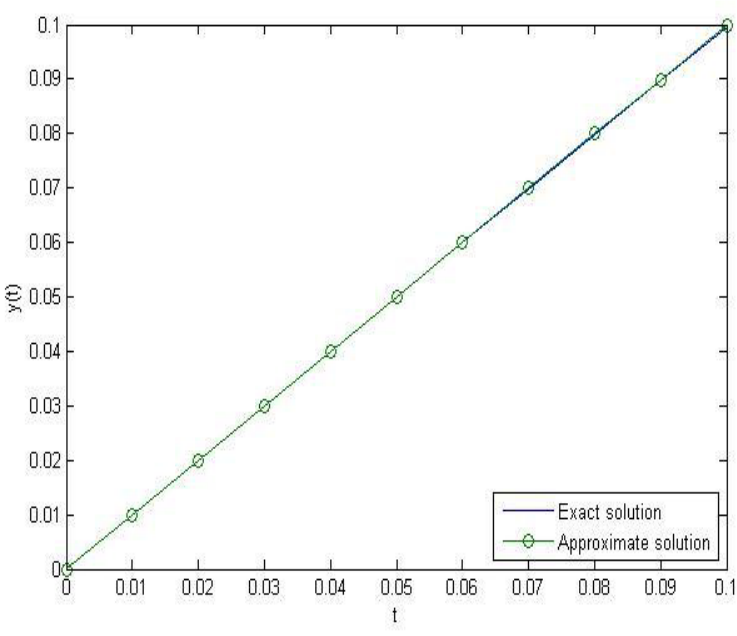

Figure 2. Comparaison of exact and approximate solution for Example2.

Table 2.and Figure 2.show that the numerical solution compared with exact is very superior and absolute error laid out in the table shows that the difference between exact solution and our presented technique is very small.

Example3. Consider the another type of Quadratic RDE $[2,4,8]$

$$
y^{\prime}=1-y^{2}(t)+2 y(t), y(0)=0
$$

Following the aforesaid technique the results have been demonstrated in the Table 3 and Figure 3.

Table 3. Comparison approximate solution with Taylor series, ADM and HPM for Example3

\begin{tabular}{|c|c|c|c|c|}
\hline $\mathbf{t}$ & $\begin{array}{c}\text { Approximate } \\
\text { Solution }\end{array}$ & $\begin{array}{c}\text { Taylor Series } \\
\text { method }\end{array}$ & ADM & HPM \\
\hline 0 & 0 & 0 & 0 & 0 \\
\hline 0.01 & 0.010100585 & 0.010100330 & 0.010100330 & 0.010100330 \\
\hline 0.02 & 0.020404690 & 0.020402612 & 0.020402612 & 0.020402612 \\
\hline 0.03 & 0.030915863 & 0.030908719 & 0.030908719 & 0.030908719 \\
\hline 0.04 & 0.041637666 & 0.041620432 & 0.041620431 & 0.041620432 \\
\hline 0.05 & 0.052573672 & 0.052539435 & 0.052539435 & 0.052539435 \\
\hline 0.06 & 0.063727448 & 0.063667310 & 0.063667309 & 0.063667310 \\
\hline 0.07 & 0.075102547 & 0.075005529 & 0.075005526 & 0.075005529 \\
\hline 0.08 & 0.086702496 & 0.086555447 & 0.086555443 & 0.086555447 \\
\hline 0.09 & 0.098530785 & 0.098318300 & 0.098318293 & 0.098318300 \\
\hline 0.1 & 0.110590857 & 0.110295195 & 0.110295185 & 0.110295195 \\
\hline
\end{tabular}




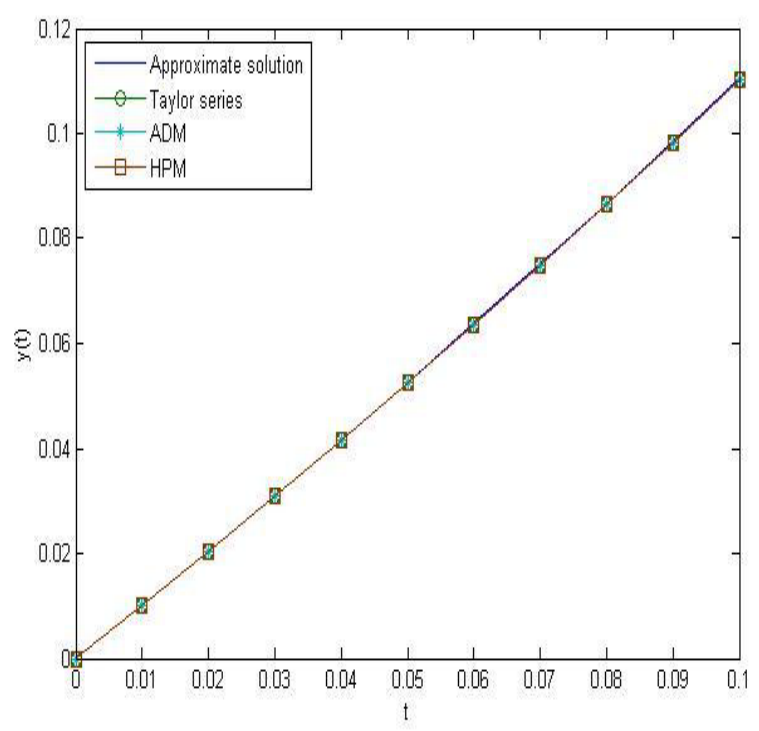

Figure 3. Comparaison of approximate solution with ADM, HPM, Taylor series.

In Table 3and Figure3, the numerical results are given for 10 values of $t$.Comparing it with ADM,HPM and Taylor series method given in [28], it is clear that the accurate value is attained as number of iteration increases.

\section{Conclusions}

In this paper a modification of Laplace transform combined with Adomian decomposition is given. The unknown function $y_{i}$ in Adomian polynomial has been replaced with the well known Newton-Raphson formula. Finally, a numerical method is constructed for solving Quadratic RDEs. The numerical results through tables and figures present that the new scheme is effective and simple in the determination of approximate solution of RDEs. All the calculations are done with MATLAB $2011 b$.

\section{References}

1. F. A. Hendi, Stud. Nonlinear Sci., 2, 129-134 (2011).

2. A. Ghorbani and S. Momani, Appl. Math. Letters, 23, 922-927(2010).

3. A. R. Vahidi, E. Babolian and Z. Azimzadeh, World Appl. Sci. J., 16, 921-925(2012).

4. J. Biazar and M. Eslami, Int. J. of Nonlinear Sci., 9, 444-447(2010).

5. O. A. Taiwo and J. A. Osilagun, Int. J. Eng. Innovat. Tech., 1, 53-56(2012).

6. T. Allahviranloo and S. S. Behzadi, Int. J. Indust. Math., 4, 389-404(2012).

7. C. Yang, J. Hou and B. Qin, WASET, 6, 569572(2012).

8. M. Khalid, M. Sultana, F. Zaidi and U. Arshad, J. Comput. Appl., 111, 1-5(2015).
9. S. Mukherjee and B. Roy, Int. J. Nonlinear Sci., 14, 251-256(2012).

10. M. Khan, M. Hussain, H. Jafari and Y. Khan, World Appl. Sci. J., 9, 13-19(2010).

11. S. Islam, Y. Khan, N. Faraz and F. Austin, World Appl. Sci. J., 8, 1100-1105(2010).

12. S. A. Khuri, J. Appl. Math., 1, 141-155(2001).

13. J. Ahmad, F. Hussain and M. Naeem, AJMP, 5, 115(2014).

14. F. K. Yin, W. Y. Han and J. Q. Song, Int. J. Appl Phys. Math., 3, 98-102(2013).

15. Y. Lin and C. K. Chen, Rom. Journ. Phys. ,59, $443-$ 453(2014).

16. N. Singh and M. Kumar, Math. Theory Model., 2, 11-22(2011).

17. J. B. Yindoula, P. Youssouf, G. Bissanga, F. Bassono and B. Some, Int. J. Appl. Math. Res., 3, 30-35(2014).

18. C. Yang and J. Hou, J. Info. Comput. Sci., 10, 213222(2013).

19. S. P. Yan, H. Jafari and H. K. Jassim, Adv. Math. Phy., 1-7(2014).

20. A. Kumar and R. D. Pankaj, Int. Scholarly Res. Netw., 2012, 1-5(2012).

21. M. A. Mohamed and M. S. Torky, Am. J. Comput. Math., 3, 175-184(2013).

22. W. Al-Hayani, Appl. Math., 4, 882-886(2013).

23. J. Fadaei, Appl. Math. Sci., 5, 1307 - 1315(2011).

24. J. A. S. Cano, IJRRAS, 16, 168-175(2013).

25. M. A. Koroma, S. Widatalla, A. F. Kamara and C. Zhang, WASET, 7, 525-529(2013).

26. N. Doğan, Math. Comput. Appl., 17, 203-211(2012).

27. A. M. Wazwaz, Appl. Math. \& Comput. , 216, 13041309(2010).

28. S. Abbasbandy, J. Comput. Appl. Math., 207, 5963(2007).

29. B. Batiha, Int. J. Appl. Math. Res., 4, 24-29(2015). 